\title{
OBTURACIÓN DE SEGUNDA MOLAR INFERIOR CON CONDUCTO EN FORMA DE "C" UTILIZANDO LAS TECNICAS THERMAFIL $Y$ GUTTAFLOW: REPORTE DE CASO
}

\author{
OBTURATION OF SECOND LOWER MOLAR WITH "C" SHAPED ROOT CANAL USING \\ THERMAFIL AND GUTTAFLOW TECHNIQUES: CASE REPORT
}

\author{
Julio Cesar Romero Gamboa ${ }^{1 a}$ Juvenal Gonzales León de Peralta²b
}

\begin{abstract}
RESUMEN
Los conductos en forma de "C" son una variación anatómica vista principalmente en segundos molares inferiores. Tienen una amplia variedad de anastomosis y comunicaciones irregulares que pueden interferir en la obturación tridimensional del sistema de conductos radiculares. Se presenta el caso de una paciente femenina de 51 años de edad que acude a la Especialidad de Carielogia y Endodoncia de la Universidad de San Martin de Porres, con inflamación en el fondo de surco sin trayecto fistuloso a nivel de la pieza 3.7, caries oclusomesial y aparente compromiso pulpar. Al examen clínico y radiográfico se confirma el diagnóstico de necrosis pulpar. Se realiza el tratamiento de conductos y en la fase de obturación se asoció una técnica mixta de los sistemas Thermafil ${ }^{\circledR}$ y Guttaflow.
\end{abstract}

Palabras clave: Microfiltración; sellado; obturación.

\section{ABSTRACT}

"C" shaped root canals are mainly an anatomycal variation of second molar. They have a wide variety of anastomosis, and irregular communications networks, which can interfere with the three-dimensional obturation of the root canal system. The case of a female patient aged 51, was admitted to the Specialized Clinical Dentistry, Faculty of Dentistry, University of San Martin de Porres in the Specialty of Endodontics and Carielogy; with inflammation of the sulcus without fistulous tract at the level of tooth 3.7 with oclusomesial caries and apparent pulpar compromise. At the clinical and radiographic examination Pulp Necrosis diagnosis is confirmed. Canal treatment is done and in the final phase, a mixed technique of obturation associated Thermafil® and GuttaFlow® systems.

Keyword: Microfiltration; sealing; obturation.

\footnotetext{
${ }^{1}$ Universidad de San Martin de Porres.

${ }^{2}$ Universidad Nacional Mayor de San Marcos.

a Especialista en Carielogía y Endodoncia.

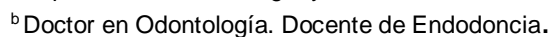

Correspondencia:

Julio Romero Gamboa.

Correo electrónico: jromerog@hotmail.com
}

Este es un artículo Open Access distribuido bajo la licencia Creative Commons Atribución-NoComercialCompartirlgual 4.0

Citar como: Romero Gamboa JC., Gonzales León de Peralta, J. Obturación de segunda molar inferior con conducto en forma de "C" utilizando las técnicas Thermafil y Guttaflow: Reporte de caso. KIRU. 2017;14(1):73-79.

https://doi.org/10.24265/kiru.2017.v14n1.10 


\section{INTRODUCCIÓN}

El conducto en forma de "C" es una variación anatómica que fue reportada por primera vez por Cooke y Cox en 1979 y, en su mayoría, es visto en los segundos molares mandibulares. Tiene especiales características anatómicas, tales como raíz fusionada con un surco longitudinal en el medio de la raíz, hacia lingual o vestibular; un piso de la cámara pulpar profundo con una apariencia anatómica infrecuente y como principal característica, la presencia de uno o más istmos conectando los conductos mesiales y distal.

La compleja anatomía de los conductos en forma de "C" requiere un procedimiento de limpieza y conformación apropiado, así como una técnica de obturación modificada.

Las áreas irregulares de un conducto en forma de "C" pueden alojar remanente de tejido o detritus infectados que pueden escapar a la limpieza y conformación. Al- Fouzan ${ }^{(1)}$ consideró que el conducto en forma de " $\mathrm{C}$ " tiene todo el contorno general en forma de $\mathrm{C}$ y que su raíz presenta una ranura longitudinal en la superficie.

Por lo descrito anteriormente, algunos autores propusieron técnicas modificadas para manejar estos casos. Los desafíos van desde el diagnóstico hasta la instrumentación, obturación y la preparación del espacio para el perno. La siguiente presentación de caso clínico tiene como objetivo general describir una técnica mixta de obturación de un conducto radicular en forma de "C".

Thermafil $^{\circledR}$ (DentsplyMaillefer, Konstanz, Alemania) es un sistema de obturación de conductos con gutapercha caliente. Consiste en un vástago central flexible de plástico recubierto con una capa de gutapercha en fase alfa (Figura 1).

El vástago de plástico sirve como un dispositivo de aplicación y debe ejercer presión ligera dentro del conducto. Una vez insertado permanece dentro del conducto radicular después de la obturación. El obturador debe ser calentado para suavizar la gutapercha antes de la inserción en el conducto. La temperatura de calentamiento y el tiempo requerido están regulados por el uso de un horno especial Thermaprep Plus ${ }^{\circledR}$ (Figura 2).

El objetivo del sistema es lograr una obturación tridimensional fiable y en un menor tiempo que las técnicas de obturación convencionales.
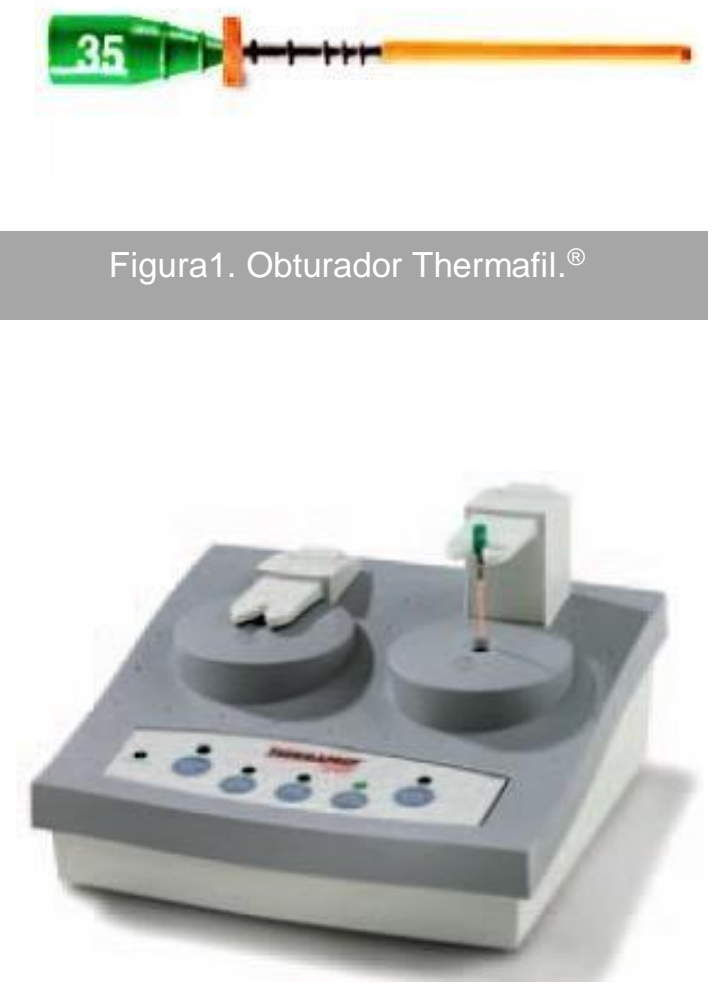

\section{Figura 2. Horno ThermaPrep Plus. ${ }^{\oplus}$}

GuttaFlow $^{\circledR}$ es un sistema frío y fluido para la obturación de conductos radiculares que combina, en un solo producto, sellador y gutapercha. Se trata de una matriz de polidimetilsiloxano con un alto contenido en polvo de gutapercha.

Ofrece una buena propiedad de sellado. Combina las propiedades del sellador y la gutapercha. Está formado por dos componentes mezclados homogéneamente en una cápsula de mezcla. Contiene polvo fino de gutapercha que se distribuye de forma homogénea después de la mezcla. Fluye fácilmente en los canales laterales y en los túbulos dentinales, sin eugenol, radioopaco, sin contracción, ligera expansión y altamente biocompatible. 

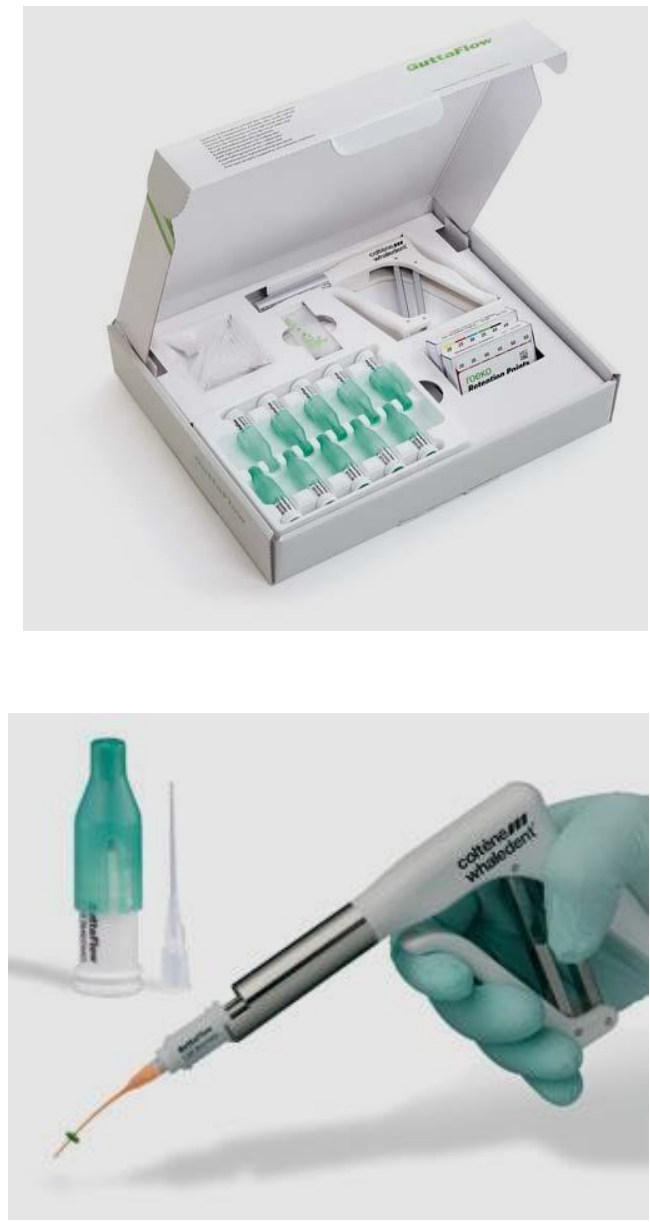

Figura 3. Sistema Guttaflow

\section{REPORTE DE CASO CLÍNICO}

Se presenta el caso de una mujer de 51 años de edad, que acudió a la Especialidad de Carielogía y Endodoncia, en la Clínica Especializada en Odontología de la Facultad de Odontología de la Universidad de San Martin de Porres, con motivo de consulta para "aliviar el problema de mis dientes".

La paciente presentaba una caries oclusomesial en la pieza 3.7, la cual le generaba molestias debido al empaquetamiento de comida y el mal aliento que esto producía. La historia médica refiere aparente buen estado general de salud y localizada y orientada en tiempo, espacio y persona. En su historia dental reveló tratamientos previos odontológicos sin complicaciones como exodoncias, restauraciones y profilaxis dental.

Al examen clínico se observó tejidos blandos en buen estado, higiene oral deficiente, piezas dentarias restauradas, presencia de caries y cálculo dental. Se apreció la migración de la pieza 3.7 al espacio dejado por la ausencia de la pieza 3.6. En la zona problema se observó una inflamación del fondo de surco sin trayecto fistuloso. La pieza 3.7 presentaba caries oclusomesial con aparente compromiso pulpar. Se realizó prueba de vitalidad pulpar al frío utilizando un spray refrigerante a una temperatura de $-50^{\circ} \mathrm{C}$ (ENDO-FROST ${ }^{\circledR}$ ), la cual resultó negativa. A la palpación y percusión mostró sintomatología y una movilidad grado I.

Radiográficamente, se observó imagen radiolúcida extensa a nivel coronal de la pieza 3.7 compatible con caries dental. Se apreció también una pequeña imagen radiolúcida a nivel apical de la pieza 3.7, una sola raíz, cámara pulpar reducida y conductos atrésicos. Se observa un ensanchamiento del espacio periodontal y una imagen radiolúcida a nivel cervical y mesial de la pieza 3.7 compatible con una reabsorción vertical de la cresta ósea.

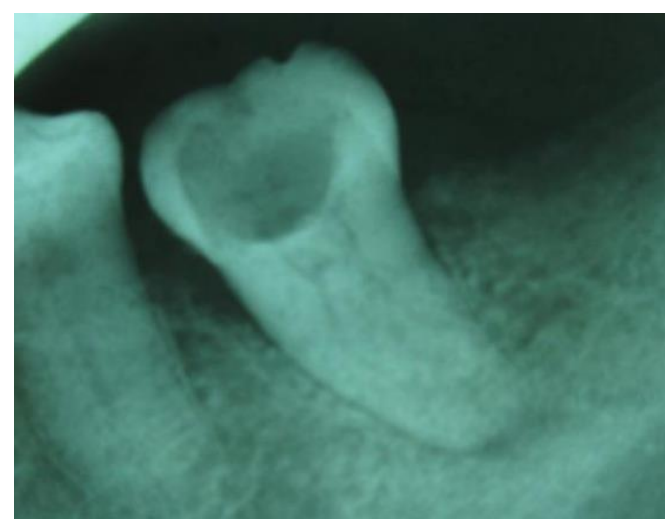

Figura 4. Radiografía inicial de diagnóstico de la pieza 3.7.

Por los signos y síntomas documentados, se confirma el diagnóstico de necrosis pulpar de la pieza 3.7. El tratamiento a seguir es la necropulpectomía, con un pronóstico reservado.

Se inició el tratamiento de conductos de la pieza 3.7 colocando el aislamiento absoluto (dique de goma, portadique Sauber ${ }^{\circledR}$ y clamp № 7A). Se empezó a remover la caries dental para luego realizar la apertura cameral con fresa redonda de diamante grano mediano y finalmente el biselado con fresa Endo $Z^{\circledR}$. Se encontró un acumulo de pulpolitos en la cámara pulpar que fueron removidos con ayuda de ultrasonido y cureta pequeña de dentina. Al realizar la limpieza de la cámara pulpar, se evidenció la particular anatomía en forma en "C" que esta tenía. Posteriormente, se realizó el desgaste compensatorio con fresa Endo $Z^{\circledR}$. 


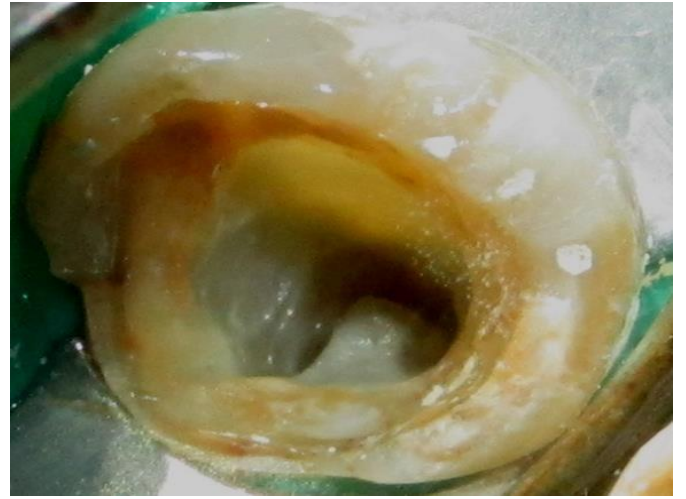

Figura 5. Apertura cameral pieza 3.7. Se aprecia la cámara y conducto en forma de "C".

Se comprobó la permeabilidad de los conductos con limas de serie especial \#08 y \#10. Teniendo el acceso listo, se irrigó con un lavado de hipoclorito de sodio al 2,5\% (solución de Labarraque), y se procedió a la limpieza y desinfección del tejido pulpar. Se determinó la longitud de trabajo a $1 \mathrm{~mm}$ del ápice radiográfico mediante la conductometría, obteniendo las siguientes medidas: conducto distal: $19 \mathrm{~mm}$., conducto mesiovestibular: $19 \mathrm{~mm}$., y conducto mesiolingual: $20 \mathrm{~mm}$.

Se realizó la preparación biomecánica para la conformación y configuración de los conductos con limas manuales y rotatorias (ProTaper $^{\circledR}$ Denstply Maillefer) mediante la utilización de la técnica híbrida de instrumentación teniendo como tope apical en los conductos mesiales $0,35 \mathrm{~mm}$, y en el conducto distal $0,40 \mathrm{~mm}$. Para la instrumentación del tercio coronal se empleó la lima Sx.

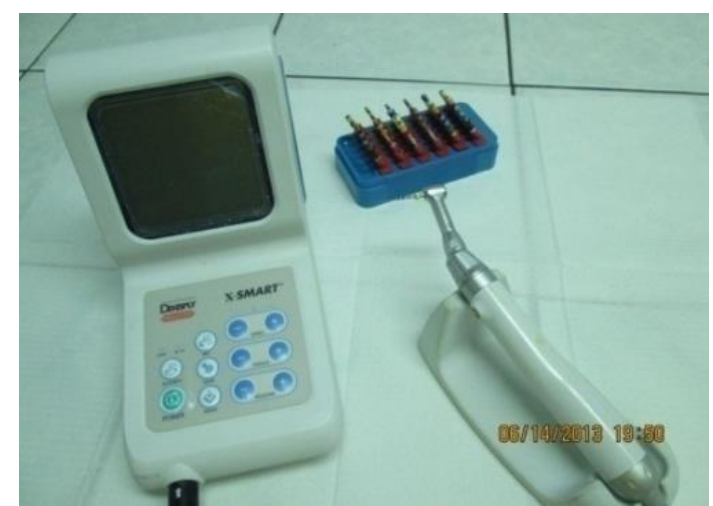

Figura 6. Motor endodóntico X- Smart. ${ }^{\circledR}$

Durante la instrumentación de los conductos se irrigó copiosamente con hipoclorito de sodio al 2,5\% con una jeringa de $20 \mathrm{cc}$ con aguja de $27 \mathrm{G}$ después de cada instrumentación. Se secaron los conductos con conos de papel estériles y se colocó con léntulos una pasta de hidróxido de calcio con suero fisiológico y paramoclofenol alcanforado (biodinámica 20ml) como medicación intraconducto por una semana. La cavidad de acceso fue sellada con cemento óxido de zinc eugenol. La siguiente cita se evaluó clínicamente. La paciente no refirió sintomatología en dicha pieza. Se reabrió el acceso cameral y se removió la medicación intraconducto remanente con irrigación abundante de hipoclorito de sodio al $2,5 \%$.

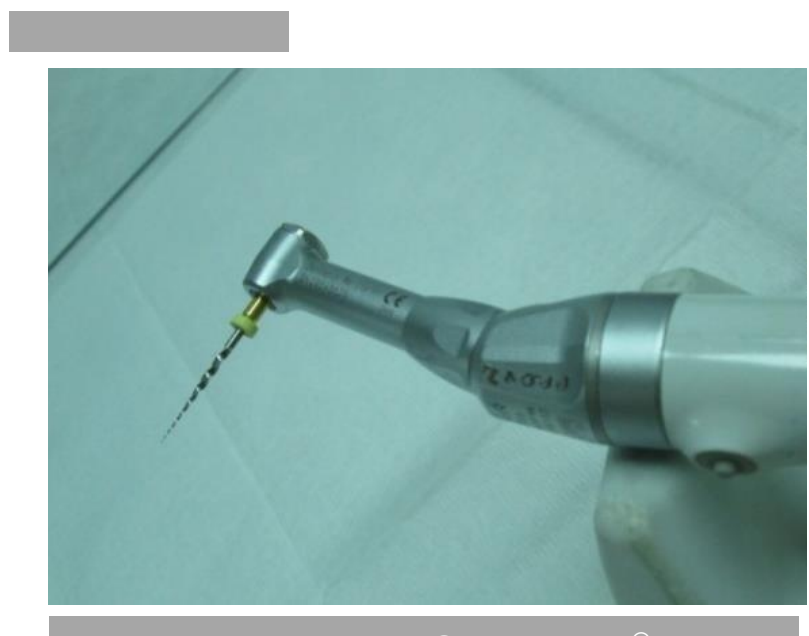

Figura 7. Lima SX Protaper. ${ }^{\circledR}$

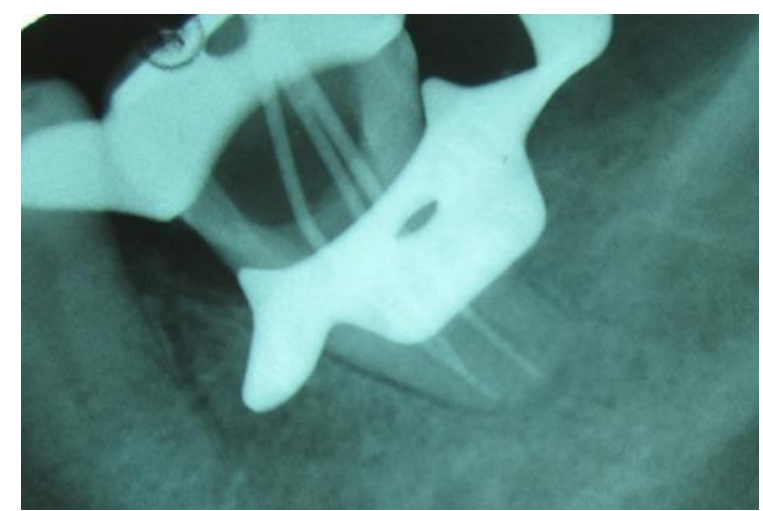

Figura 8. Conometría en pieza 3.7.

Finalmente, se utilizó como irrigación final EDTA al $17 \%$ por 30 segundos. Luego se neutralizó con suero fisiológico, se secó con conos de papel estériles y se procedió a obturar los conductos. Para la obturación de los conductos mesiales se utilizó la técnica de obturación Thermafil ${ }^{\circledR}$, usando los 
obturadores \#35; mientras que en el conducto distal se utilizó la técnica GuttaFlow ${ }^{\circledR}$ y condensación
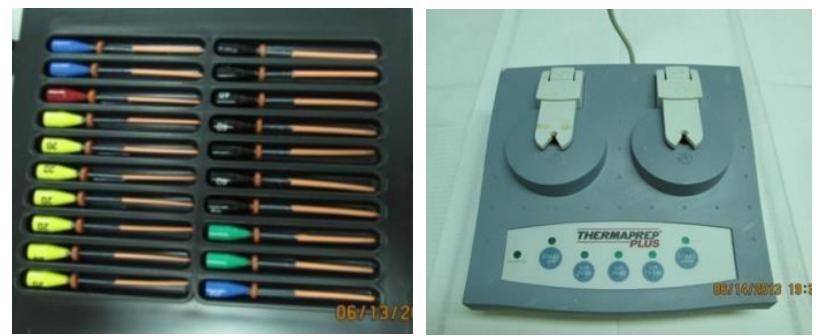

Figura 9.HornoThermaPrep Plus. ${ }^{\circledR}$ Figura 10. Obturadores Thermafil. ${ }^{\circledR}$

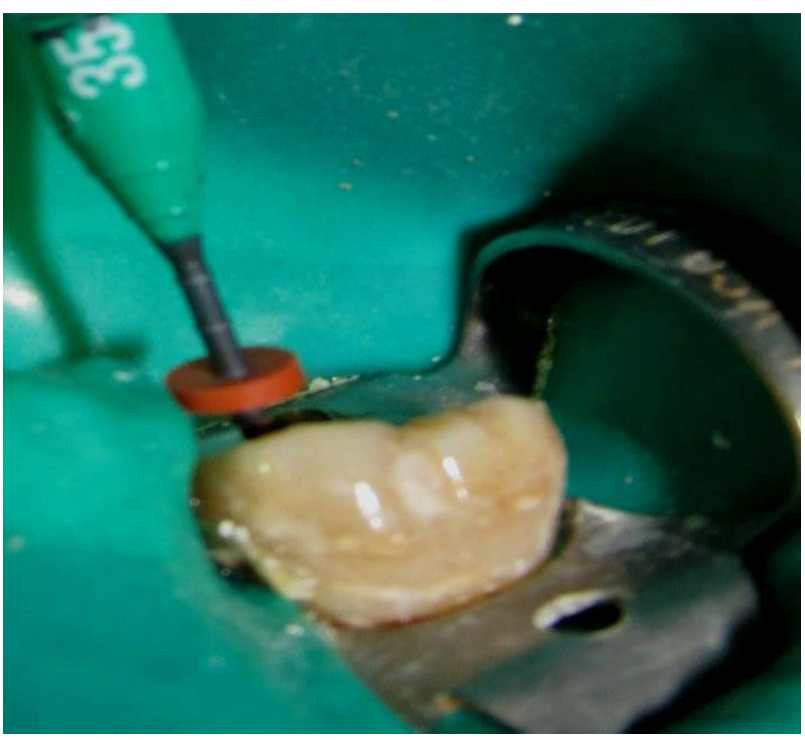

Figura 11. Colocación de obturador Thermafil.

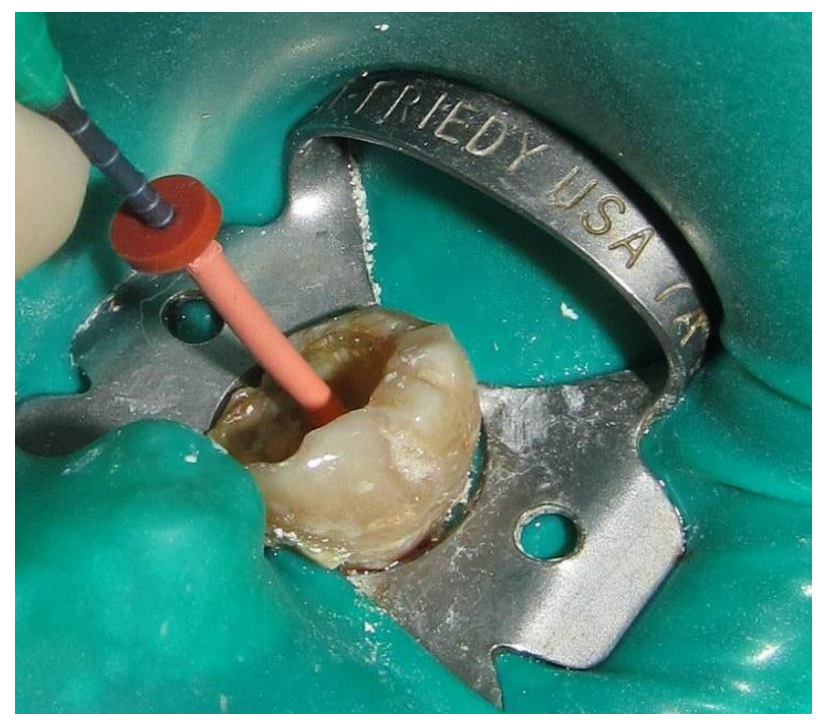

Figura 12. Obturador en conducto mesial lateral con conos accesorios. En ambas técnicas se empleó el cemento sellador de Grossman ${ }^{\circledR}$.

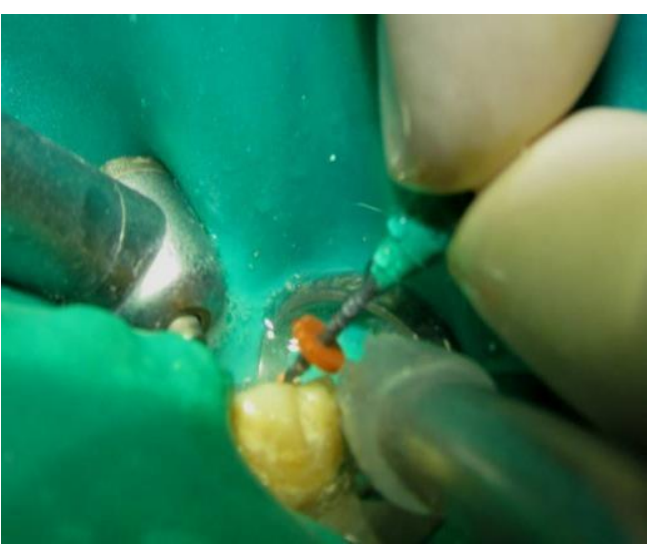

Figura 13. Corte del vástago con una fresa

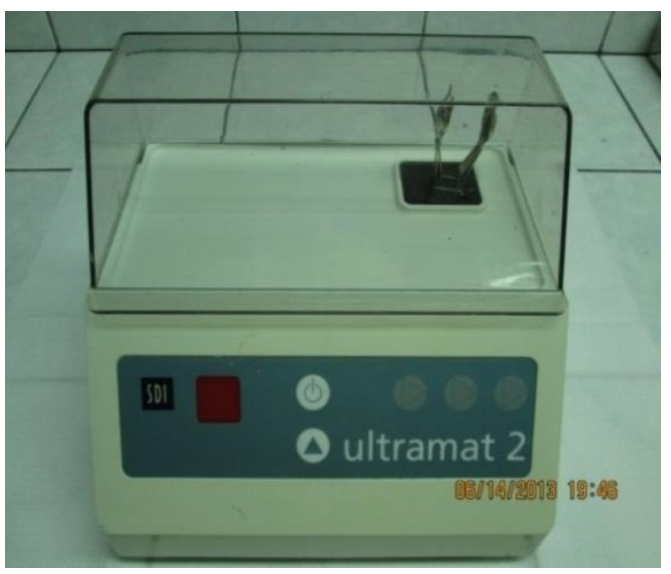

Figura 14. Amalgamador

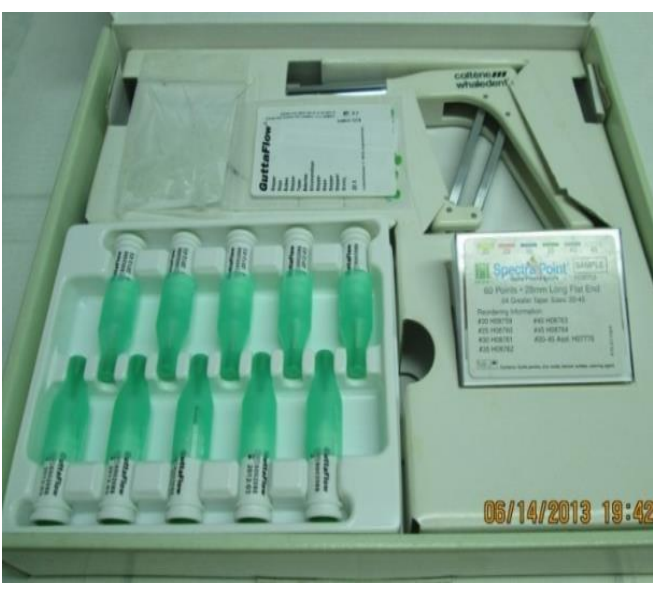

Figura 15. Sistema Guttaflow. ${ }^{\circledR}$ 


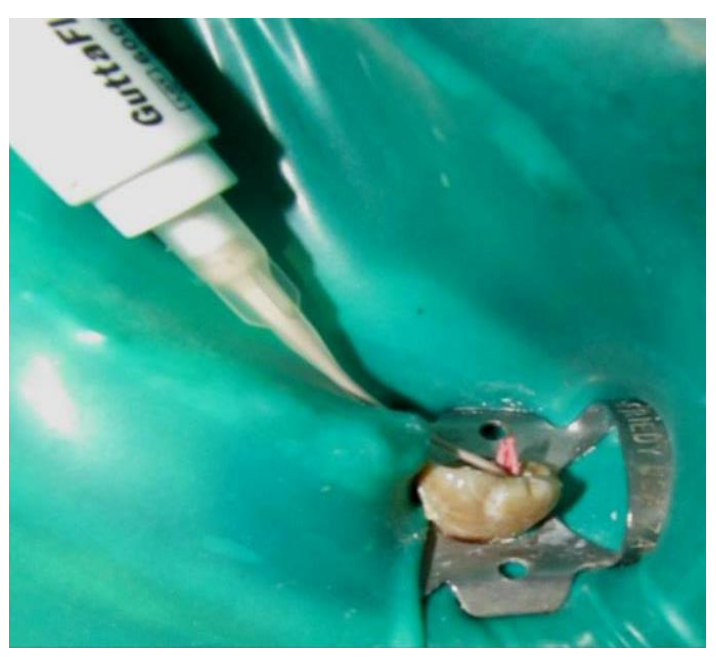

Figura 16. Inyección del Guttaflow. ${ }^{\circledR}$

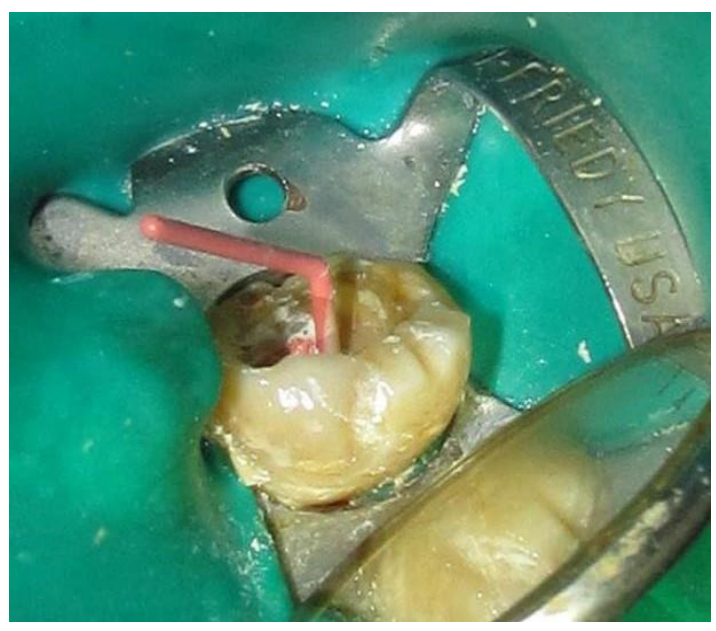

Figura 17. Colocación del cono maestro

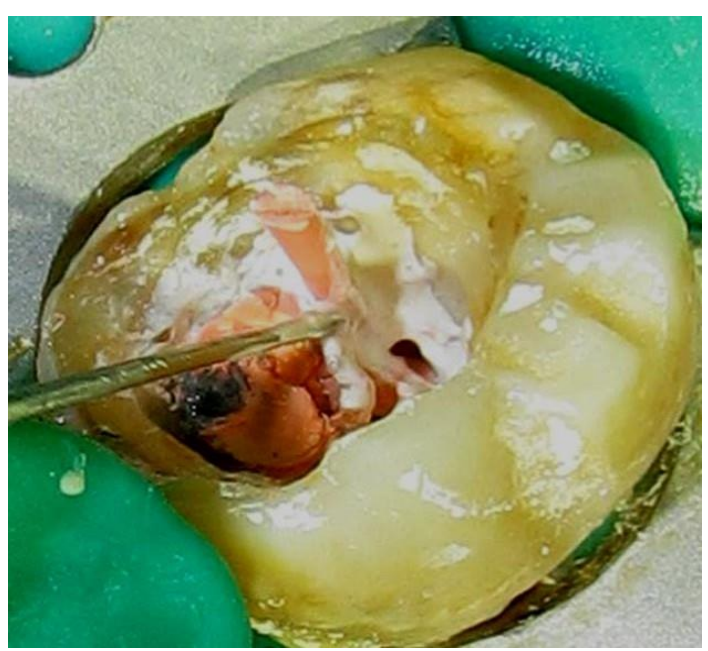

Figura 18. Colocación de conos

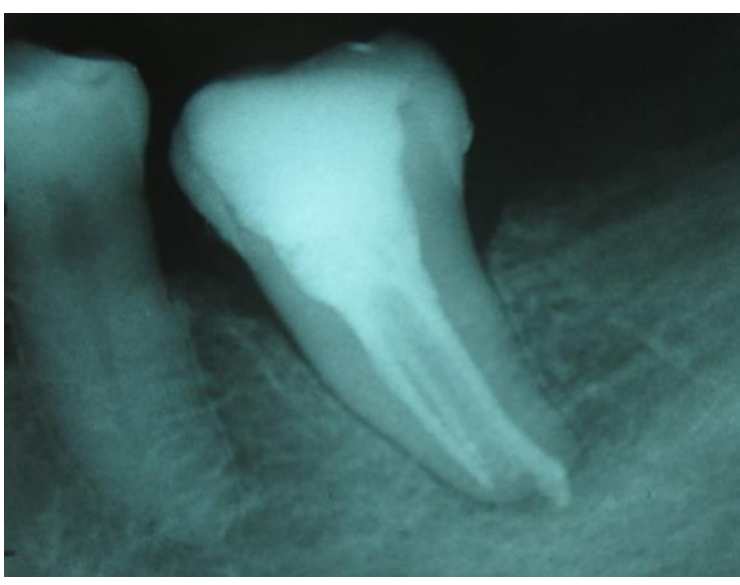

Figura 19. Una semana de finalizar el tratamiento.

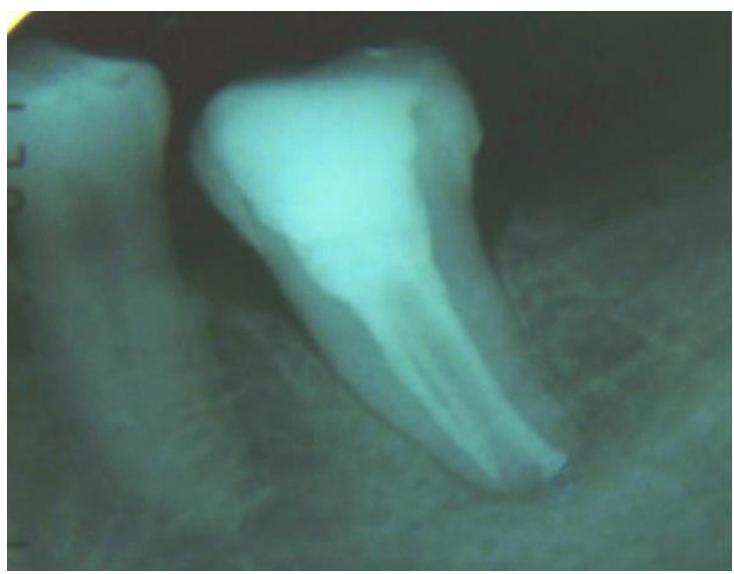

Figura 20. A los nueve meses de finalizar el tratamiento.

Se realizaron controles radiográficos a distancia a la semana, mes y nueve meses, mostrando reparación periapical y reabsorción del material que había sido extravasado apicalmente. Clínicamente, la paciente presentaba silencio clínico, sin presencia de tumefacciones y tejidos periodontales circundantes en estado saludable

\section{DISCUSIÓN}

Existen pocos reportes clínicos en los cuales se sugiera una secuencia ideal para la obturación de los conductos radiculares en forma de "C"; $\sin$ 
embargo, la mayoría de autores concluye que la mejor técnica es aquella en la cual se emplea la gutapercha termoplastificada.

Uno de estos autores es Walid(2), que en el 2000 sugirió obturar los conductos principales utilizando la técnica de condensación lateral, y la parte central del conducto con Obtura System mediante dos condensadores. Él argumentaba que las áreas divergentes del conducto en " $C$ ", que con frecuencia no tienen forma, pueden ofrecer resistencia al flujo de material de obturación; así como también los istmos que comunican los conductos principales a través de los cuales puede pasar el material de obturación de un conducto a otro; por consiguiente, las fuerzas hidráulicas disminuirán considerablemente, lo que podría comprometer seriamente la calidad de la obturación. En este caso, la colocación de dos conos maestros y el bloqueo de la entrada del conducto con un condensador aumentan la resistencia hacia el paso de material de obturación de un conducto a otro. Esta medida genera más presión hidráulica y permite que el material de obturación fluya a otras áreas del conducto en $\mathrm{C}$, generando una obturación hermética.

Otro reporte fue el presentado por Kumar et al..$^{(3)}$ en el 2001, en el cual empleó gutapercha quimioplastificada para la obturación de conductos en forma de " $\mathrm{C}$ ", usando trementina blanca rectificada como disolvente. En contraste con su estudio anterior, Kumar et al. ${ }^{(4)}$ en el 2011 emplearon únicamente la técnica de condensación lateral y obtuvieron excelentes resultados en la obturación del conducto en "C", y emplearon como cemento sellador el AH Plus.

Miglani et al.(5) en el 2002 reportó el uso de ultrasonido para facilitar la eliminación de detritus entre los istmos, usando la irrigación ultrasónica con $1,26 \%$ de hipoclorito de sodio y $17 \%$ de EDTA, alternativamente, para eliminar la capa de barrillo y para mejorar la penetración de la gutapercha termoplastificada. En la obturación utilizó el sistema Thermafil, que usa gutapercha en fase alfa con bajo peso molecular, la cual sufre menos cantidad de contracción y es más adecuada para obturar las distintas variaciones anatómicas en los conductos radiculares, con resultados satisfactorios.

Tanto la técnica clínica empleada en el manejo del conducto radicular en forma de "C" como los resultados obtenidos después del tratamiento endodóntico tienen semejanza con el estudio hecho por Carpegna ${ }^{(6)}$ en el 2013, quien luego de instrumentar los conductos radiculares procedió a obturarlos mediante el empleo de la técnica Thermafil. Las imágenes radiográficas mostraron una distribución adecuada de la gutapercha termoplastificada con sellado apical homogéneo. Durante el seguimiento aparecieron todos los casos libres de sintomatología y mostraron salud perirradicular. Se concluye así que al igual que en el presente reporte de caso clínico, la técnica de obturación con el sistema Thermafil es fácil de usar y fiable en el tratamiento de conductos radiculares en forma de " $C$ ".

Según la bibliografía consultada se concluye que la presencia de los sistemas radiculares en forma de " $C$ " en segundas molares inferiores es poco frecuente, y presenta un desafío al endodoncista debido a su compleja anatomía.

El correcto diagnóstico clínico, el reconocimiento temprano de estas configuraciones mediante distintas tomas radiográficas y la exploración clínica intracameral facilitan la limpieza, conformación y obturación de estos sistemas de conductos.

La mayoría de autores consultados concluyeron que las técnicas de obturación con gutapercha termoplastificada son las más usadas para el tratamiento de los sistemas de conductos en forma de "C".

La asociación de las técnicas de obturación Thermafilß y GuttaFlow ${ }^{\circledR}$, utilizadas en el presente caso clínico, facilitaron el rellenado de toda la compleja anatomía del sistema de conductos radiculares en forma de "C".

\section{REFERENCIAS BIBLIOGRÁFICAS}

1. Al-Fouzan K. C-shaped root canals in mandibular second molars in a Saudi Arabian population. Int Endod J 2002; 35: 499-504.

2. Walid N. The use of two pluggers for the obturation of an uncommon C-shaped canal. J Endod 2000; 26: 422-424.

3. Kumar K, Bhat K. C-shaped mandibular molarObturation by chemoplasticized gutta-percha technique. Endodontology 2001; 13: 27-30.

4. Kumar K, Mathur S, Shrivastava R, Srivastava R. Conventional root canal therapy of C-shaped mandibular second molar-A Case report. Int $\mathrm{J}$ Contemp Dent 2011; 2(5): 151-153.

5. Miglani S, Bharadwaj $T$, Velmurugan $N$, Parameswaran A. C-shaped Canals. Endodontology 2002; 14: 66-68.

6. Carpegna G, Rigolone M, Pasqualini D, Berutti E.Cshaped management with Thermafil system. En: $32^{\circ}$ Congresso Nazionale Vincitore Premio Giorgio Lavagnoli; 2013. 